\title{
A Rare Association of Congenital Diaphragmatic Hernia with Lower Esophageal Atresia and Perforation
}

\author{
Narendra Kumar Are, K. Nagarjuna, and Lavanya Kannaiyan \\ Department of Paediatric Surgery, Niloufer Hospital for Women and Children, Osmania Medical College, Hyderabad 500 017, India \\ Correspondence should be addressed to Narendra Kumar Are, naren_are@yahoo.com
}

Received 20 May 2010; Accepted 15 June 2010

Academic Editor: Frans J. Walther

Copyright ( $) 2010$ Narendra Kumar Are et al. This is an open access article distributed under the Creative Commons Attribution License, which permits unrestricted use, distribution, and reproduction in any medium, provided the original work is properly cited.

Congenital diaphragmatic hernia is known to be associated with esophageal atresia, which is a rare association. We report a rare occurrence of congenital diaphragmatic hernia and lower esophageal atresia.

\section{Introduction}

Abnormalities of the esophagus such as gastroesophageal reflux disease (GERD), esophageal motility disorders, esophageal duplications cysts, and tracheoesophageal fistula with esophageal atresia are rare but documented occurrences with congenital diaphragmatic hernia $[1,2]$. We report a rare association of lower esophageal atresia with congenital diaphragmatic hernia $(\mathrm{CDH})$.

\section{Case Report}

A 10-day male child presented with respiratory distress without cyanosis since birth. On clinical examination, patient had tachypnea, with a scaphoid abdomen. The persistent drooling of saliva led to the suspicion of esophageal atresia. A red rubber catheter was passed into the esophagus, but there was resistance at $15 \mathrm{~cm}$ from the alveolar margin. Chest X-ray showed evidence of left $\mathrm{CDH}$ with mediastinal shift and the tip of nasogastric tube at the level of the diaphragm. A contrast esophagogram was done which showed holdup of dye at the level of the diaphragm (Figure 1). With the suspicion of associated esophageal obstruction and $\mathrm{CDH}$, a laparotomy was done using a chevron incision. The operative findings include left posterolateral $\mathrm{CDH}$, complete disruption of the esophagogastric junction with a blindending esophagus, and a sealed esophageal perforation at the esophagogastric junction (Figure 2).
The surgical correction included repair of $\mathrm{CDH}$ and esophagogastric anastomosis with a feeding jejunostomy. The postoperative course was uneventful. Jejunostomy feeds were started on the 4 th postoperative day. Contrast esophagogram was done on the 10th postoperative day. It showed free flow of dye into the stomach. At discharge, the child was on full oral feeds. He has been followed up for 3 months. The child's general condition is good with adequate weight gain.

\section{Discussion}

Esophageal anomalies are known to be associated with $\mathrm{CDH}$. These associations include tracheoesophageal fistula with esophageal atresia, GERD, esophageal dysmotility, esophageal duplication cysts, and esophageal ectasia [1, 2]. The possible noted explanations include the kinking of the esophagogastric junction [2] and abnormal innervation of the esophagus by the vagus and recurrent laryngeal nerve [3].

Tracheoesophageal fistula with esophageal atresia is a known association of $\mathrm{CDH}$, albeit a rare one with an incidence of 0.005 per 1000 births [4]. In this case report, there is atresia of the lower esophagus at the esophagogastric junction. It appears to be an acquired event probably due to vascular compromise by the acute kinking of the stomach in the thorax because of the $\mathrm{CDH}$. In an extensive review of the English literature, there is only one similar case reported 


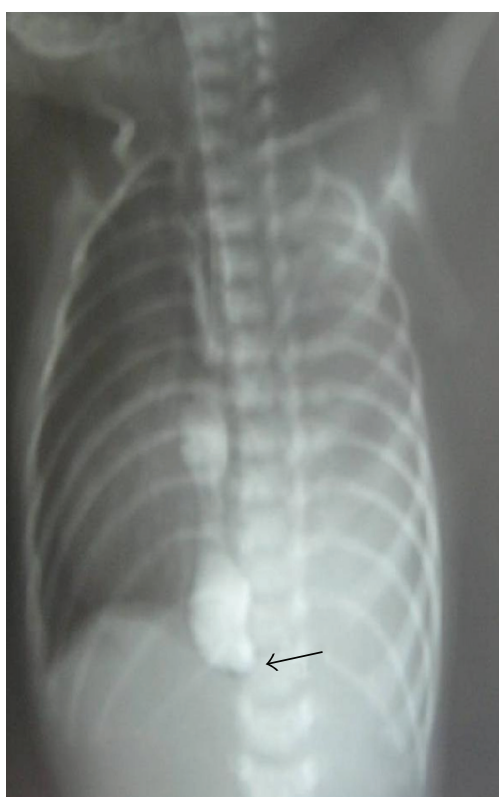

FIGURE 1: Contrast esophagogram showing holding up (arrow) of contrast at the level of the diaphragm with evidence of $\mathrm{CDH}$ with lower esophageal obstruction.

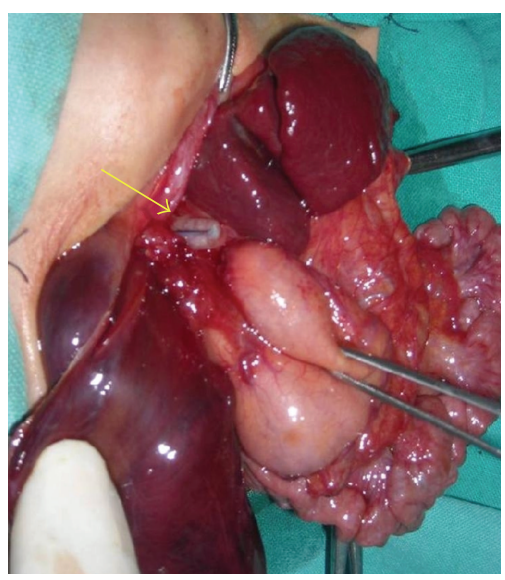

FIgURE 2: Operative photograph showing a blind ending of the lower esophagus with a sealed perforation (arrow).

by van Dooren et al. [5], where there was an atresia of the lower end of the esophagus that was not suspected until the postoperative period. In this case report, there was preoperative diagnosis of a lower esophageal obstruction; hence surgery was done using a chevron (rooftop) incision. This incision allows access for correction of both of the lesions. A feeding jejunostomy is a useful adjunct until the restoration of the esophagogastric continuity.

In conclusion, esophageal anomalies are known to occur with $\mathrm{CDH}$. Lower esophageal atresia is a rare association and a preoperative suspicion of this association, clinically, leads to timely correction of both of the lesions. A cheveron incision gives good access to correct both the $\mathrm{CDH}$ and lower esophageal atresia.

\begin{abstract}
Abbreviations
$\mathrm{CDH}$ : Congenital diaphragmatic hernia

GERD: Gastroesophageal reflux disease.
\end{abstract}

\section{Acknowledgments}

The authors acknowledge the contribution of Dr. Leela Kumar, Dr. Hariprasad, and Dr. K. Srinivas in the management of the patient and preparation of the manuscript.

\section{References}

[1] I. Karnak, M. E. Şenocak, F. C. Tanyel, and N. Büyükpamukçu, "Abnormal esophageal anatomy associated with a congenital diaphragmatic hernia: report of a case," Surgery Today, vol. 31, no. 11 , pp. 1005-1007, 2001.

[2] C. J. H. Stolar, J. P. Levy, P. W. Dillon, C. Reyes, P. Belamarich, and W. E. Berdon, "Anatomic and functional abnormalities of the esophagus in infants surviving congenital diaphragmatic hernia," American Journal of Surgery, vol. 159, no. 2, pp. 204207, 1990.

[3] L. Martínez, S. González-Reyes, E. Burgos, and J. A. Tovar, "The vagus and recurrent laryngeal nerves in experimental congenital diaphragmatic hernia," Pediatric Surgery International, vol. 20, no. 4, pp. 253-257, 2004.

[4] H. Takehara, N. Komi, A. Okada, M. Nishi, and K. Masamune, "Left diaphragmatic hernia associated with lower esophageal atresia," Pediatric Surgery International, vol. 8, no. 4, pp. 339340, 1993.

[5] M. van Dooren, D. Tibboel, and C. Torfs, "The cooccurrence of congenital diaphragmatic hernia, esophageal atresia/tracheoesophageal fistula, and lung hypoplasia," Birth Defects Research Part A, vol. 73, no. 1, pp. 53-57, 2005. 


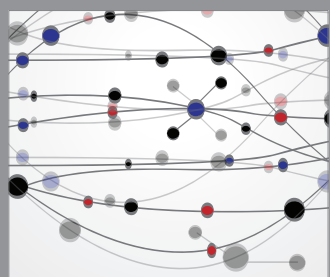

The Scientific World Journal
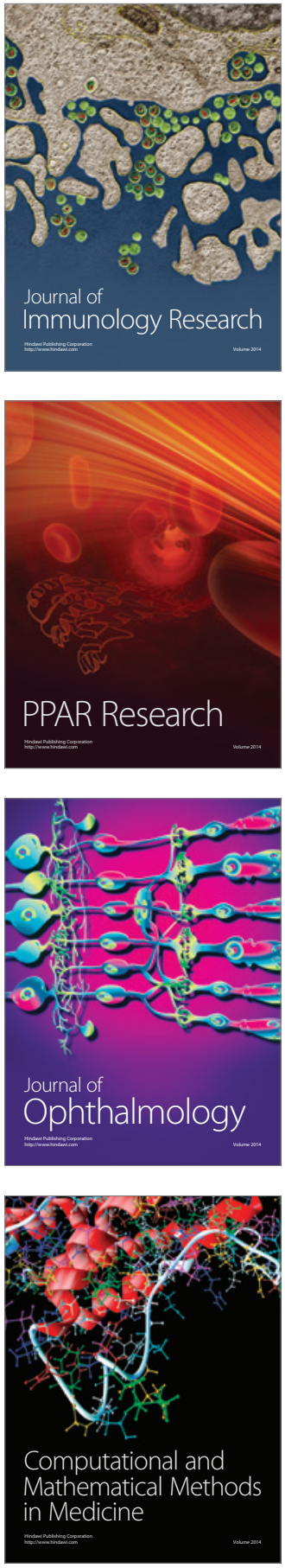

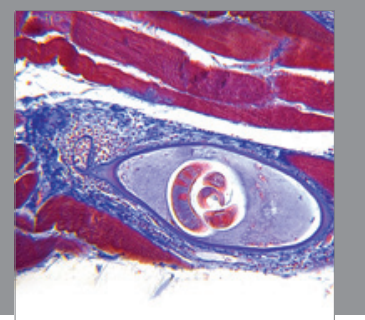

Gastroenterology

Research and Practice
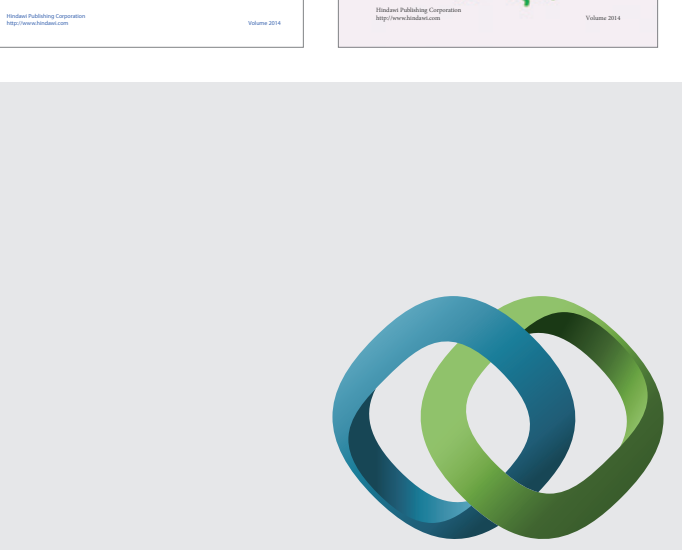

\section{Hindawi}

Submit your manuscripts at

http://www.hindawi.com
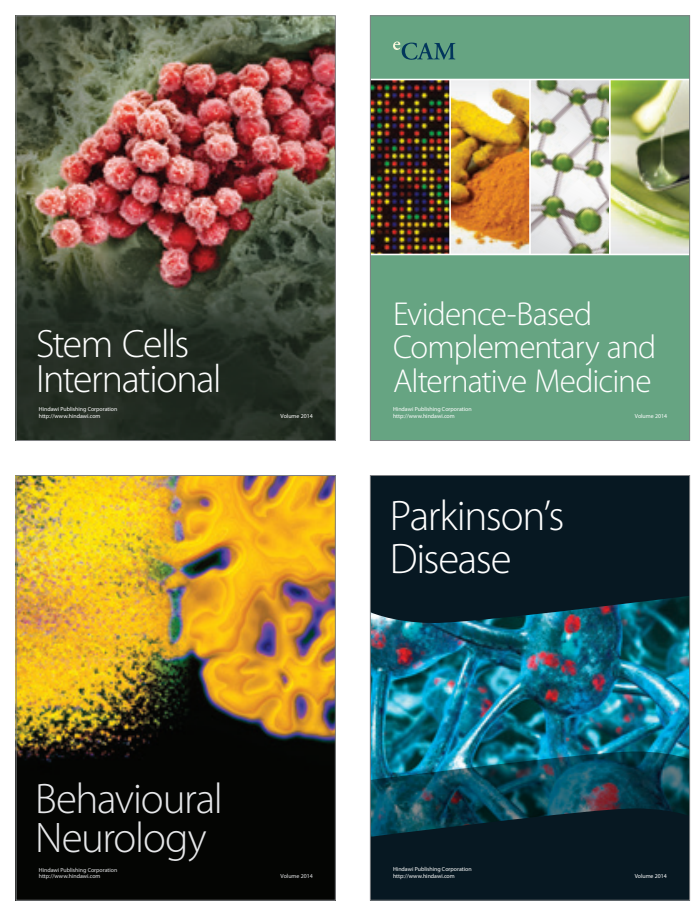

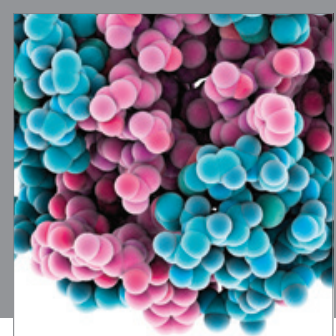

Journal of
Diabetes Research

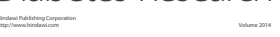

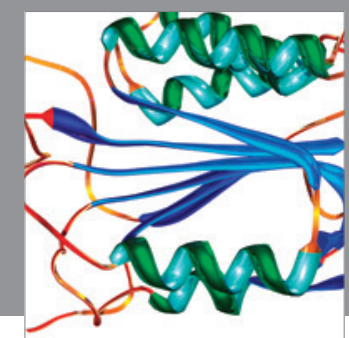

Disease Markers
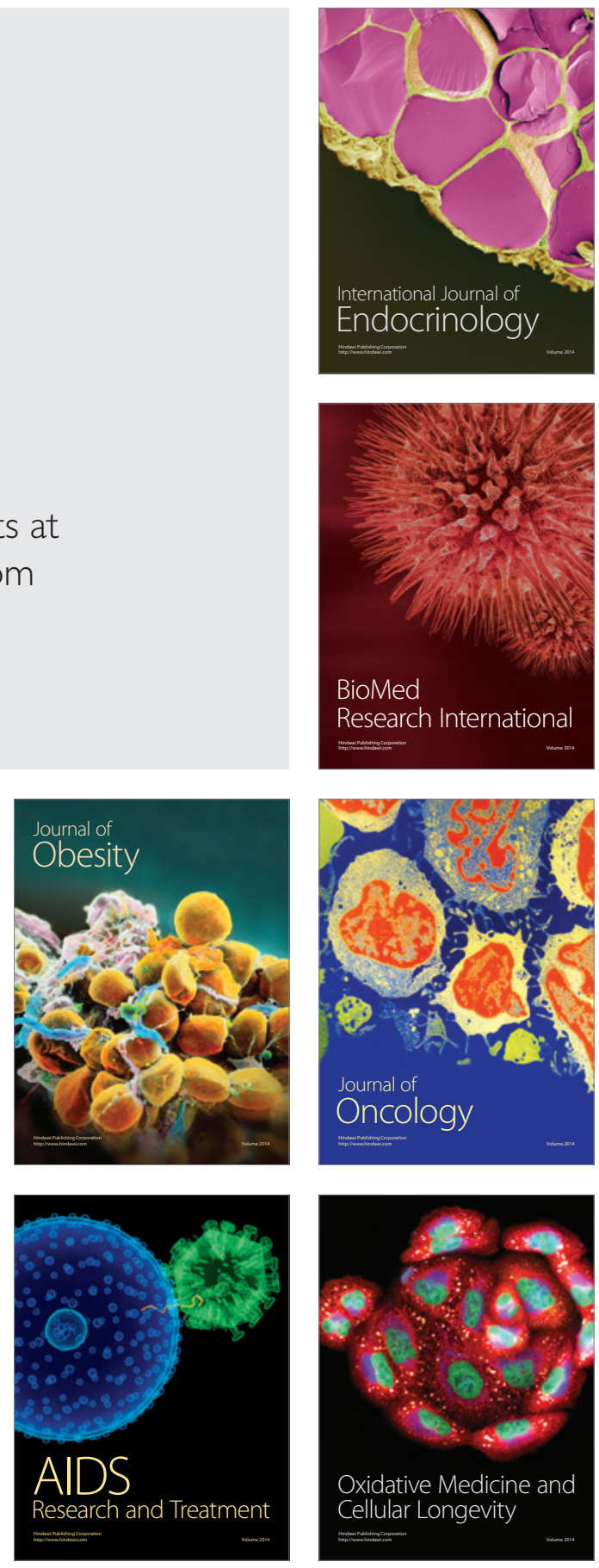